\title{
I tassi di complicanze postoperatorie diminuiscono con l'invecchiamento del chirurgo (!)
}

\author{
Gianlorenzo Dionigi ${ }^{1,2}$
}

Accettato: 30 agosto 2021 / Pubblicato online: 3 marzo 2022

c) The Author(s) 2022, corrected publication 2022

Commento a:

\section{Relation between surgeon age and postoperative} outcomes: a population-based cohort study. R. Satkunasivam, Z. Klaassen, B. Ravi, K.-H. Fok, T. Menser, B. Kash, B.J. Miles, B. Bass, A.S. Detsky, C.J. Wallis.

\section{Can Med Assoc J (2020) 192(15):E385-E392}

Come è noto, gli interventi chirurgici di alta specializzazione anche in ambito endocrinochirurgico richiedono una profonda conoscenza, esperienza, capacità di decisione clinica e professionalità tecnica. L'età può essere dannosa per le capacità cognitive e motorie, ma l'età è anche associata all'esperienza. I risultati dello studio finora disponibili sulla relazione tra l'età del chirurgo e l'esito postoperatorio del paziente sono contraddittori $[1,2]$.

Nello studio di Satkunasivam e collaboratori è stata esaminata la misura in cui l'età del chirurgo, tenendo conto del volume dell'attività chirurgica, influisce sull'esito postoperatorio del paziente. Si tratta di uno studio di coorte retrospettivo condotto con pazienti adulti che avevano procedure di emergenza o elettive convenzionalmente aperte o laparoscopiche effettuate in Ontario, Canada dal 2007 al 2015. Sono comprese anche le procedure di chirurgia endocrina. L'associazione tra età del chirurgo (29-80 anni), volume e esito postoperatorio negativo a 30 giorni (i.e., morte, complicanze, riammissione ospedaliera) è stata analizzata su base multivariata. Le procedure selezionate per ciascuna sottodisciplina chirurgica comprendevano la chirurgia generale, endocrinochirurgia, cardiochirurgia, neurochirurgia, ecc.

$\triangle$ G. Dionigi

gianlorenzo.dionigi@unimi.it

1 U.O. Chirurgia Generale, Istituto Auxologico Italiano IRCCS, Milano, Italia

2 Dipartimento di Fisiopatologia Medico-Chirurgica e dei Trapianti, Università di Milano, Milano, Italia
I chirurghi di età $>65$ anni avevano un volume di casi più elevato rispetto ai chirurghi di età $<65$ anni, e hanno anche praticato più frequentemente in un contesto accademico [3]. In generale, il 12\% dei pazienti ha avuto morbidità postoperatorie. Con l'aumentare dell'età del chirurgo, i tassi complessivi di outcome e tutti e tre i parametri analizzati (tassi di morte, complicanze e riammissione) sono diminuiti continuamente. Ad ogni intervallo di 10 anni di età del chirurgo, i tassi di outcome negativi esaminati sono diminuiti del $5 \%$. La differenza di esito tra chirurghi $>65$ anni $(5 \%$ di tutti i chirurghi, $6 \%$ di tutti gli interventi) e età $<65$ anni era indipendente dalla complessità degli interventi ed era significativa per entrambe procedure chirurgiche in emergenza ed elettive.

Gli autori concludono che i chirurghi più anziani potrebbero essere più in grado di fornire indicazioni bilanciate per il rischio postoperatorio. Tuttavia, ammettono anche che le procedure selezionate per l'analisi potrebbero spiegare le differenze rispetto a studi simili pubblicati finora. Gli autori concludono che i loro risultati parlano contro i regolamenti formali di pensionamento per i chirurghi. I chirurghi più anziani che possono continuare a svolgere lavori altamente qualificati dovrebbero essere incoraggiati a continuare il loro lavoro.

Funding Note Open access funding provided by Università degli Studi di Milano within the CRUI-CARE Agreement.

Open Access This article is licensed under a Creative Commons Attribution 4.0 International License, which permits use, sharing, adaptation, distribution and reproduction in any medium or format, as long as you give appropriate credit to the original author(s) and the source, provide a link to the Creative Commons licence, and indicate if changes were made. The images or other third party material in this article are included in the article's Creative Commons licence, unless indicated otherwise in a credit line to the material. If material is not included in the article's Creative Commons licence and your intended use is not permitted by statutory regulation or exceeds the permitted use, you will need to obtain permission directly from the copyright holder. To view a copy of this licence, visit http://creativecommons.org/licenses/by/4. $0 /$. 


\section{Bibliografia}

1. Dionigi G, Boni L, Rovera $F$ et al (2008) Defining the learning curve for video-assisted thyroidectomy. Int J Surg 6(Suppl 1):S1-3

2. Dionigi G, Bacuzzi A, Boni L et al (2008) What is the learning curve for intraoperative neuromonitoring in thyroid surgery? Int J Surg 6(Suppl 1):S7-12

3. Satkunasivam R, Klaassen Z, Ravi B et al (2020) Relation between surgeon age and postoperative outcomes: a population-based cohort study. Can Med Assoc J 192(15):E385-E392
Nota della casa editrice Springer Nature rimane neutrale in riguardo alle rivendicazioni giurisdizionali nelle mappe pubblicate e nelle affiliazioni istituzionali. 